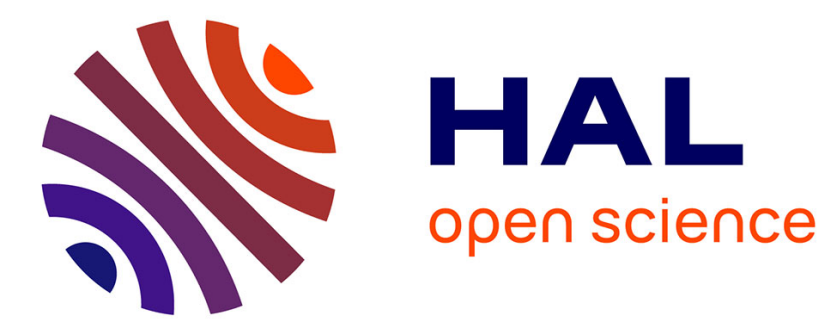

\title{
Une urbanité de confrontation
}

Jérôme Boissonade

\section{- To cite this version:}

Jérôme Boissonade. Une urbanité de confrontation. Espaces et sociétés (Paris, France), 2006, 126, pp.35-52. hal-00840660

\section{HAL Id: hal-00840660 https://hal.science/hal-00840660}

Submitted on 4 Jul 2013

HAL is a multi-disciplinary open access archive for the deposit and dissemination of scientific research documents, whether they are published or not. The documents may come from teaching and research institutions in France or abroad, or from public or private research centers.
L'archive ouverte pluridisciplinaire HAL, est destinée au dépôt et à la diffusion de documents scientifiques de niveau recherche, publiés ou non, émanant des établissements d'enseignement et de recherche français ou étrangers, des laboratoires publics ou privés. 

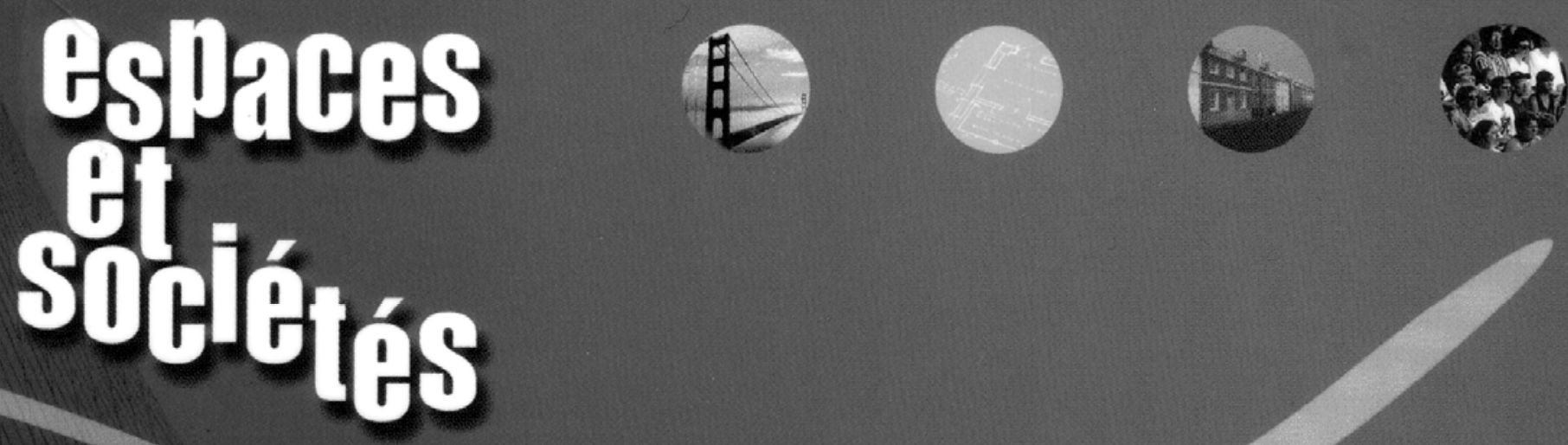

Las lienx

des liens sociaux

érès

126 


\section{Une urbanité de confrontation Regroupements de jeunes et gestionnaires de l'espace urbain ${ }^{1}$}

\section{Jérôme Boissonade}

$\mathbf{E}_{\text {n }}$ deçà de la figure du « citoyen », acteur collectif idéal d'une participation républicaine, mais qui est souvent attendu en vain par les acteurs urbains, « l'habitant » a remplacé le «salarié » comme objet légitime des politiques publiques. Tel Janus, il possède une face compassionnelle, celle de l'exclu, objet des démarches de réparation, et une face obscure, celle du jeune délinquant, objet des entreprises sécuritaires.

Aujourd'hui considérée comme un délit, « l'occupation des halls d'immeuble par les jeunes » cristallise les contradictions propres à cette figure. Pourtant, le sens que prennent les regroupements d'adolescents ne peut se réduire à ce lieu emblématique, au désœuvrement et aux combines évoqués de façon récurrente, ou à une violence sans objet. Une cage d'escalier, un

Jérôme Boissonade, maître de conférences en sociologie, université du Littoral (ULCO) et chercheur à l'Institut des mers du Nord et l'IPRAUS.

jboisson@msh-paris.fr

1. Cet article s'appuie sur un travail d'enquête mené auprès de gestionnaires de l'espace social et urbain de Bobigny, Créteil et Nanterre, ainsi que sur une observation participante de plusieurs rassemblements de jeunes dans deux cités de Créteil. 
banc, une allée ou un espace commun peuvent être, le matin, un lieu de rencontre où des personnes âgées attendront le facteur, l'après-midi un terrain de jeu pour les enfants et le soir venu, un espace de rassemblement pour les plus grands. Dans la même cité, les relations peuvent être courtoises à un endroit et conflictuelles dans un autre. Les lieux concernés peuvent changer au cours de l'année. Loin de tout déterminisme, les mêmes jeunes peuvent paraître affables ou menaçants suivant les occasions. On peut tenter de mieux comprendre ces paradoxes en considérant l'urbanité d'un espace, moins par son statut (la place publique, le centre commercial...), ou par la composition des publics qui le fréquentent (civils ou incivils), qu'à partir des « situations » qui font se croiser lieux et liens sociaux. Sans dénier l'intérêt des deux premières approches, nous allons nous intéresser ici aux situations et aux processus de catégorisation, plus qu'à des types d'espaces ou de personnes. Ceci peut permettre, nous semble-t-il, de dépasser certaines prénotions qui sont attachées à ces types, et notamment le sens essentiellement anomique donné aux tensions publiques qui émaillent l'ordinaire des regroupements de jeunes.

Hors des cités, les tensions urbaines, sont le plus souvent considérées comme la conséquence d'expressions légitimes, par exemple celles qui peuvent naître à l'occasion d'une manifestation de rue. Ces expressions et ces tensions sont reconnues comme un gage de la «bonne santé » de l'espace public. Pourtant, le rassemblement de jeunes, côtoyé par les autres publics, paraît être une situation quotidienne tout aussi pertinente pour mettre en évidence certaines transformations de l'urbanité contemporaine. "L'hospitalité de confrontation »(Joseph, 1998a : 103), que nous allons travailler, remet notamment en cause les procédures habituelles de catégorisation induites par toute interaction urbaine.

Après quelques précisions sur notre approche méthodologique, les contextes et les situations concernés, nous montrerons en quoi le rôle de ces regroupements s'apparente à celui joué par les espaces intermédiaires décrits par L. Roulleau-Berger (1991). En partant du constat d'une urbanité relative aux situations, nous nous attarderons sur les processus de catégorisation mis en œuvre, puis sur les mécanismes socio-ethnologiques qui différencient ces situations de rassemblement des autres modes de relations sociales au sein des cités HLM. Notre but est de mettre en évidence l'actualité de cette « urbanité de confrontation », au-delà des ensembles d'habitat social.

\section{ENJEUX ET LIMITES DES DIFFÉRENTS PARADIGMES}

Une approche socio-ethnologique permet de discerner deux paradigmes explicatifs des liens développés par les agrégations juvéniles dans les lieux qu'ils occupent.

Le premier paradigme interroge ces regroupements dans une optique régulatrice, s'appuyant sur des recherches formulées en termes de « désorga- 
nisation », sur l'expérience des gestionnaires des espaces urbains (bailleurs, concierges, services techniques...) et sur les « remontées » du terrain. Cette conception dominante, relayée par les médias ${ }^{2}$, s'incarne dans des problématiques de " groupe » ou de «bande ». Les liens qui se déploient à travers ces formes d'agrégation sont le plus souvent analysés en termes de déviance (anomie, inversion de la domination...), de territoire (appropriation, milieu...) et d'identité (délinquante, adolescente...). Les espaces qui les reçoivent sont décrits comme étant des lieux de l'entre-soi et des motifs de conflit avec les autres publics. Cette conception souligne la nécessité et la vulnérabilité de l'espace public.

Le second paradigme interroge ces pratiques juvéniles dans une approche compréhensive. Dans ce courant disciplinaire, les liens sociaux qui traversent ces pratiques sont analysés en termes de risque (mesurable, assurable...), de confiance (nécessaire, partagée...) et de compétences (acquises, intégratrices...). Les espaces qui les reçoivent sont décrits comme étant des espaces ressources et des lieux de recomposition, s'inspirant en droite ligne des travaux de l'École de Chicago. Cette conception souligne la nécessité et la vulnérabilité des solidarités qui sont à l'œuvre.

Les enjeux des situations de rassemblement sur les espaces communs en habitat collectif (extérieurs et intérieurs), semblent résulter de ces deux nécessités : celles d'espaces publics efficients et de nouvelles solidarités. Ces deux exigences sont abordées le plus souvent en termes de mixité (résidentielle) et de mobilité.

L'approche en termes de mixité résidentielle repose sur un idéal d'équilibre socio-ethnique, préalable à l'émergence d'un véritable espace public. Dans cette vision cohésive et stabilisée de la République, chaque aire urbaine se doit d'être représentative de la diversité de la population dans son ensemble. Or, l'objectif de mixité, s'il crédibilise une obligation de moyens pour les gestionnaires de l'espace urbain, repousse toujours à plus tard la réalisation d'un espace public pratique dans lequel les publics puissent s'éprouver les uns les autres.

L'approche en termes de mobilité repose sur un idéal d'accessibilité, préalable à l'émergence d'une véritable solidarité. Dans cette vision faisant fi des dominations et des logiques structurales, cette accessibilité se doit de permettre à tous d'activer des liens (professionnels, affinitaires...). Or, l'accélération des mobilités, si elle multiplie les liens faibles, accélère aussi les ségrégations en permettant à ceux qui le peuvent de se retrouver entre eux et de fuir la différence. L'accessibilité se retourne alors en détachement.

2. On peut noter à ce propos le rôle central que joue l'INHES (ex-IHESI), organisme de recherche dépendant du ministère de l'Intérieur et sa revue Les Cahiers de la sécurité intérieure, dans la diffusion de cette conception régulatrice des rapports sociaux, notamment à travers le concept d'incivilité. 
Les limites des deux paradigmes (régulation et compréhension) ne remettent pas en cause cette double nécessité d'espace public et de solidarité. Toutefois, elles nous conduisent à préciser l'approche méthodologique, les contextes et les situations que nous allons retenir pour aborder les espaces de rassemblement de jeunes et leur traversée par les différents publics.

\section{APPROCHE, CONTEXTE, SITUATION : TROIS DIFFICULTÉS À SURMONTER}

« Ne vous plaignez pas que l'espace soit pris par des mômes qui vous posent problème, parce que vous le laissez ».

Ces paroles d'un responsable local de la Politique de la ville, s'adressent aux habitants d'une cité. Elles révèlent la sensibilité de cet enjeu partagé des liens aux lieux pour les différents publics qui vivent ou traversent ces espaces.

La première difficulté, pour comprendre ces rapports aux lieux, est de tenir compte des contextes qui sont très divers d'un quartier à l'autre. Dans une même ville, les cités qui sont « la banlieue de la banlieue », "plutôt village » ou celles dont « les limites floues favorisent une plus grande perméabilité des groupes » (gardien[ne]s d'immeuble, A...), relèvent de problématiques en partie différentes, ici périphérique, là enclavée, ou encore urbaine.

La deuxième difficulté de toute étude socio-spatiale est de sortir des approches déterministes, couramment utilisées par les acteurs :

« Ce sont des jeunes qui généralement habitent là, qui ont peut-être vécu un certain moment ensemble, les familles peut-être se connaissent. Et il y a cette espèce d'appartenance à une communauté, qui n'est plus la communauté maghrébine, française, ceci, cela, mais la communauté de destin, de la cage d'escalier, de l'immeuble » (responsable de club de prévention, C...).

Il s'agit ici au contraire de montrer la complexité des situations suivant les publics, les espaces et les moments.

La troisième difficulté lorsque l'on s'intéresse à ces espaces, est de faire la part de ce qui relève d'activités délictueuses, et notamment du trafic de drogue, qui implique un rapport aux lieux bien particulier, et de ce qui s'explique par d'autres motifs de regroupement ${ }^{3}$ :

«Les groupes se font et se défont, sauf pour ceux qui font des conneries. Car ceux-là sont devenus des copains plutôt par obligation, parce qu'ayant fait des conneries ensemble, plus on reste ensemble et moins il y a de risque de se faire attraper. Alors que si quelqu'un disparaît du groupe ou se retrouve seul dans une structure isolée, on aurait plutôt peur qu'il balance les autres » (responsable de club de prévention, C...).

3. Bien entendu, cette distinction relève avant tout d'un procédé heuristique. 
Malgré leur statut spécifique, ces regroupements liés au trafic « domestique $^{4}$ » prennent place parmi les autres rassemblements, essentiellement liés à l'âge des enfants et des adolescents. Les jeunes qui les composent s'autorisent des migrations de l'un à l'autre suivant les moments de la journée. Cependant, le travail d'observation montre rapidement que la vente de drogue requiert une présence stable pour être présent au moment opportun, sur un lieu choisi à dessein :

«Les dealers se mettent sous le bâtiment F, sous le hall avec les pilotis, parce que comme ça, ils ne peuvent être ni photographiés, ni filmés depuis un appartement, et ils ont une vue côté place et côté allée » (responsable centre social, C...).

En revanche, l'existence du trafic change de nature, lorsqu'il s'opère à grande échelle :

"Cette cité est acquise à la grosse criminalité (belles voitures, gros receleurs, gros bonnets) parce qu'on peut dégager rapidement sur la A 86. Sur le quartier, il n'y a pas d'amicale de locataires et pas de service public. [...] Tout le monde connaît le trafic. Tous les habitants le voient. C'est là où les grosses cylindrées viennent, c'est ici qu'ils livrent. C'est le lieu de livraison et ça se dispatche sur le reste du quartier » (coordonnateur de quartier, évoquant la même cité, C...).

La présence d'un trafic lourd est donc fortement liée à la désertion de l'espace commun par la population, alors que la revente domestique, tend au contraire à fixer les revendeurs sur place.

Ces précisions concernant l'approche déterministe, les contextes urbains et les situations de trafic sont importantes, parce qu'elles nous permettent de travailler maintenant d'autres figures sociologiques que celles habituellement convoquées pour traiter du «phénomène des banlieues ». Intéressons-nous maintenant à la diversité des situations socio-spatiales ordinaires que rencontrent quotidiennement les jeunes qui participent aux rassemblements ou les côtoient. Si les principales figures du lien aux lieux sont étroitement liées à la question des conflits, elles montrent d'abord l'émergence d'une urbanité relative aux situations rencontrées.

\section{DE L'ACCOUTUMANCE À L'ESPACE INTERMÉDIAIRE : VERS LA CONSTRUCTION D'UNE URBANITÉ RELATIVE}

La première figure du lien au lieu qui se détache est celle de «l'accoutumance » :

4. Nous caractérisons ainsi la revente de drogue sur l'espace public (haschich, cannabis, ecstasy et crack pour l'essentiel), qui concerne une clientèle limitée de consommateurs. Les reventes, en amont et en aval, s'opèrent de manière plus discrète. Elles font intervenir des flux et des réseaux, soit commerciaux (« grossistes »), soit affinitaires (fêtes, amis...). 
« Un bâtiment a dû être démoli parce que les hourdis étaient dangereux. [...] Après la démolition, les gens dispersés dans leurs nouveaux logements se retrouvent quand même à stationner sur le lieu de leur ancien bâtiment démoli où ceux de 12-16 ans jouaient avant au ballon et où il y avait un jeu de boules » (responsable organisme HLM, B...).

À travers le concept de mémoire collective, M. Halbwachs (1968) a montré la résistance dont peut faire preuve un groupe à l'égard des transformations urbaines, que ce soit par le maintien de ses habitudes antérieures ou la reconfiguration du nouveau lieu sur le modèle de l'ancien. Les prises d'espace se font, en effet, dès le plus jeune âge. Les lieux de rassemblement retenus évoluent pour se rapprocher progressivement des espaces les plus passants de la cité (Boissonade, 2001). Cependant, les interventions urbaines, même de faible ampleur, modifient la forme de ces rassemblements. Elles révèlent à la fois les rapports de dépendance qui s'étaient développés et les transformations du rapport à l'espace qui s'ensuivent.

La ville de Créteil souhaitait réaliser, avant l'été, une rénovation du trottoir où est situé le rassemblement principal de la cité. Des fûts préfabriqués en béton faisant office de jardinières étaient utilisés comme appuis pour les adolescents. La chaussée était suffisamment vaste à ce carrefour pour qu'un véhicule puisse stationner, le temps d'aller acheter le journal, ou un peu plus longtemps pour un jeune désirant parader avec sa voiture. Les jardinières ont été supprimées et le trottoir élargi. Des chasse-roues canalisent la circulation et rendent tout stationnement gênant. Du coup, le rassemblement s'est légèrement dispersé sur l'ensemble du mobilier urbain environnant, barrières, poubelles, panneaux...y compris sur les nouveaux chasse-roues. Les jeunes ont notamment réinvesti un muret situé à dix mètres de là (ils l'avaient plus ou moins abandonné à la suite d'une démarche sécuritaire précédente qui avait consisté à poser une grille sur ce muret situé devant un cabinet médical). Après réalisation de ce nouvel aménagement, les adolescents sont plus mobiles et utilisent les autres lieux tout proches, de manière plus fréquente. La restructuration a mis à l'épreuve les routines des rassemblés, en les disséminant sur l'ensemble du carrefour. Plus étalé, le rassemblement est plus perméable aux rencontres avec des connaissances ou des passants. Il est, par contre, encore plus exposé et visible, et les comportements spatiaux sont moins canalisés (les jeunes échangent souvent de part et d'autre de la chaussée, n'hésitent pas à la traverser, ou à y stationner). La répartition spatiale par strate d'âge s'est renforcée. Les jardinières qui ont été enlevées et sur lesquelles les jeunes s'adossaient, ne servent plus de point d'appui au rassemblement principal, contraint de retrouver ses marques. Ce dernier joue plus difficilement son rôle de pivot des autres rassemblements (celui des petits, des moyens...). Cette déstabilisation spatiale a semble-t-il accentué la porosité des rassemblements à l'égard de leur environnement. Cependant, leur 
fragilisation a aussi altéré les relations qui existaient entre les différents rassemblements, combinant à la fois cohésion et souplesse.

Une deuxième figure des liens aux lieux se détache, celle du dépassement de cette accoutumance par les acteurs. Les liens, y compris de dépendance par rapport aux lieux, ne se posent jamais en termes " purs ». Les personnes sont toujours associées à des lieux :

« À l'autre bout du quartier, on est toujours dans une notion où personne ne se connaît parce que c'est loin et en même temps on est toujours [pour les filles maghrébines] dans cette espèce de hantise où à un moment ou à un autre : "j'ai croisé quelqu'un..." [un frère par exemple] » (responsable club de prévention, C...).

Mais inversement un lieu est toujours associé à l'action de certaines personnes, un espace est toujours en mouvement :

«Les groupes de jeunes, y compris les enfants, de la place Nationale et ceux de la place Valence, se bagarrent au moment où ils se rencontrent. Ils n'ont pas d'endroit spécifique. [...] Les zones sont plus fonction des groupes qui les occupent que des particularités "géographiques". Par exemple, avant, le marché à côté de la rue Walter était considéré comme faisant partie de la zone "place Nationale" parce que les jeunes habitant rue Walter fréquentaient énormément cette place. Maintenant, ce n'est plus le cas, alors la zone "place Nationale" est limitée à la place » (gardien d'immeuble, A...).

Ne pas renvoyer dos à dos ces deux interprétations des liens sociaux aux lieux, la première par l'espace, la seconde par les publics, implique de considérer les situations qui mettent en présence l'un et l'autre. Les publics qui se côtoient et les espaces aménagés par les gestionnaires doivent faire sens pour chacun.

Dans cette hypothèse, une troisième figure complémentaire des précédentes peut être mise en évidence, celle de " l'espace intermédiaire ${ }^{5}$ ». Cette figure de l'espace intermédiaire prend ici le sens d'un entre-deux socio-spatial qui relie le séparé et sépare le relié (Simmel, 1988 [1909]).

Au même titre que les rapports frontaliers révèlent la conception qu'une société se fait de son territoire, ces situations intermédiaires semblent capitales pour analyser les relations des liens sociaux par rapport aux lieux. Les contours mêmes des rassemblements de jeunes peuvent nous aider à mieux rendre compte de ces situations interstitielles entre les espaces et entre les publics. Ces rassemblements/espaces intermédiaires prennent en effet plusieurs formes :

5. D'abord envisagée en termes d'interstice, cette notion a été travaillée plus récemment à travers la notion de seuil et d'espace de recomposition entre centre, périphérie et espaces sociaux (Roulleau-Berger, op. cit.). 


\section{Contact}

Ici, l'espace de rassemblement est un lieu plus ou moins neutre où les participants viennent spécialement, notamment pour ne pas aller chez l'autre. Ces lieux ne sont pas retirés mais ont souvent un caractère urbain affirmé (grand espace vert, placette...), à proximité de lieux à centralité urbaine forte (services, équipements...).

\section{Passage}

Dans ce cas de figure, le rassemblement ne constitue pas un but de déplacement. Les personnes provenant y compris d'autres quartiers, traversent cet espace pour aller au collège, sur un terrain de sport ou au centre-ville. Ces points de «passage » ont une fréquentation plus imprévisible que les autres. On peut considérer, par extension, une voie de circulation comme constituant entièrement un espace de rassemblement/passage, mais c'est le plus souvent au sein d'une cité que ce rôle est davantage rempli (banc, muret, jeu pour enfant, carré de pelouse...).

\section{Seuil}

L'espace de rassemblement est ici l'endroit par où l'on passe pour sortir ou pour rentrer dans le quartier ou la cité, c'est un espace qui fonctionne toute l'année. Les rassemblements/seuils sont souvent proches des commerces pour plusieurs raisons. D'une part, ils maintiennent un flux de personnes à peu près constant qui assure la vie de ces espaces intermédiaires. D'autre part, ce sont en général les lieux les mieux desservis par les transports en commun et un repli est possible, en cas de mauvais temps, (galerie, café...) sans pour autant s'enfermer chez soi ou dans un hall.

\section{Soupape}

L'espace de rassemblement permet, dans ce cas, de sortir au quotidien de zones d'habitat qui ont la particularité d'être relativement autosuffisantes en équipements. On ne sort pas de la cité pour avoir quelque chose que l'on n'a pas chez soi, mais l'on en sort pour aller se « changer d'air » sur ces espaces intermédiaires et sortir de la pression de l'environnement résidentiel. Ce sont souvent des terrains de sport combinant parfois d'autres fonctions (jeux d'enfants...), qui jouent ce rôle parce que ce sont des structures proches qui peuvent être utilisées quotidiennement sans être pour autant « au milieu » des zones d'habitat. 


\section{Accueil}

La dernière forme que prend l'espace de rassemblement est l'espace d'accueil. Il peut s'agir d'un équipement, de ses abords, ou d'un espace situé au sein d'une cité. Les supports physiques de ces sociabilités sont bien souvent minimaux (escalier, barrière, muret, trottoir...). Les personnes viennent de plusieurs endroits pour s'y rencontrer :

« Il y a une chose que j'ai oublié de signaler, qui est très importante, c'est que l'Esprit, c'est une terre d'accueil. Combien de gens, de l'extérieur, sont venus dans ce quartier, y ont pris goût, y sont restés, et ont commencé à trâner dans les halls ? C'est impressionnant. Et toutes générations confondues. Sur ce quartier, tu as l'impression que très rares sont les personnes qui s'ouvrent sur l'extérieur, qui vont traîner... Non, on attire les gens. Et ils restent avec nous » (Ali, 24 ans).

Pour qualifier l'urbanité relative qui marque les situations de rassemblement, la figure de l'accoutumance semble montrer la dépendance des publics par rapport aux lieux. Celle du dépassement de cette dépendance met en évidence la co-construction des rapports sociaux et des lieux. La troisième figure, celle de l'espace intermédiaire, nous a permis de mieux comprendre le rapport entre dépendance et autonomie des relations sociales à l'égard des espaces qui les accueillent. D'une part, la diversité des formes d'espace intermédiaire, met en évidence le caractère relatif et « situé » de l'urbanité qui les caractérise. D'autre part, cette diversité des formes d'espace intermédiaire montre comment les relations sociales se construisent sur des situations et des espaces interstitiels en tirant parti des écarts entre les liens aux lieux des différents publics.

Les processus qui permettent de construire cette urbanité spécifique interrogent en retour certains mécanismes habituels de construction des relations sociales, et notamment, nous allons le voir, le travail quotidien de catégorisation qui permet de structurer le rapport à la situation. En poussant l'espace public dans ses retranchements, les rassemblements s'en révèlent de bons analyseurs. Ce travail de catégorisation problématise en effet les situations quotidiennes et rend crédible l'existence d'un espace public pratique.

\section{CATÉGORISER : UNE NÉCESSITÉ INTERACTIONNELLE ET UNE DIMENSION STRUCTURALE}

«Moi, j'ai habité le 1 en tant que locataire pendant trois ans. Jamais j'aurais pensé que la cité allait jusque-là. Quand on est au 1, on est sur la rue. C'est les gamins qui font que la cité se mélange » (gardienne d'immeuble, A...).

Cette réflexion d'une concierge, à propos de la petite cité dont elle a la charge, montre la déconnexion dont on peut faire preuve comme habitant, 
entre l'espace privé de son appartement et les espaces communs qui l'entourent. Cette méconnaissance tranche pourtant avec les autres récits recueillis dans la même ville, pour lesquels la « cité » est au cœur des représentations. Il est vrai que ce petit groupe d'immeubles, assez récents, s'inscrit dans une trame viaire qui le dépasse. Greffé non pas sur une voirie d'accès, mais sur une voie de circulation urbaine, la vie paraît être ailleurs, dans cette agitation de la ville que seuls les enfants et les adolescents semblent mettre à distance en investissant tous les recoins de la cité. Les « gamins » jouent donc apparemment un rôle essentiel dans les sociabilités intermédiaires entre l'espace $\mathrm{du}$ « chez soi » et l'anonymat relatif qui est la règle dans le reste de la ville. L'ex-habitante devenue concierge, n'en a véritablement pris conscience qu'avec ses nouvelles responsabilités, lui imposant la vision globale d'un espace dont elle doit dorénavant assurer le maintien. Le rôle qu'endossent les jeunes adolescents lui semble par ailleurs indispensable au «mélange » des habitants. Un «mélange », souvent réduit à la mixité par les acteurs urbains, affublé des qualités que porte la ville, par laquelle tout devient public grâce à la diversité de ceux qui la composent.

Ce modèle de l'espace public est conforme aux définitions qu'en ont donné Simmel et Habermas. Il repose sur un idéal de spontanéité de la rencontre et sur la lisibilité de celle-ci. Juger de la normalité de cette rencontre nécessite en effet de pouvoir se la rendre intelligible. Plusieurs auteurs ont travaillé sur les processus de «typification » (Schütz, 1987) ou « d'identification catégorielle » auxquels se livre chacun de nous dans ses relations à autrui, afin de lire la situation. Cependant, l'essentiel tient peut-être à ce que l'assimilation d'un individu ou d'un groupe à une catégorie ou une communauté « est de l'ordre de l'indécidable : il flotte. Ce qui signifie qu'il est de l'ordre du problématique » (Peraldi, $1989: 14)$. C'est cette dimension problématique du travail de catégorisation qui, nous semble-t-il, est au centre de l'élaboration d'un "espace public pratique », tributaire de la diversité des situations. Cette dimension problématique revêt au moins trois aspects.

Premièrement, le travail de catégorisation permet une construction identitaire par différenciation d'avec autrui et une «mise en ordre du réel » qui nous entoure :

«Ce sont toujours les mêmes lieux qui ont été occupés par le même type de personnes et de la même manière » (gardien d'immeuble, A...).

Deuxièmement, toute catégorisation rend possible un engagement individuel partageable :

«Le discours de tous, du cadre A au chômeur, c'est : "Qu'ils fassent ce qu'ils veulent, mais pas dans mon escalier" » (responsable politique de la ville, B...).

Enfin, les catégorisations fonctionnent comme objet de discussion, et plus profondément comme question politique : 
« Il y a un manque de terrains vagues. Tous les équipements (city stade...) sont spécialisés. Ils ont une fonction forcément limitée, alors qu'un terrain vague, vous imaginez quelque chose et vous y faites ce que vous avez imaginé. Personne ne vous embête parce que vous ne gênez personne et qu'il n'y a rien à casser. [...] Là, ça répondra au désir de chacun. Il faut arrêter de mettre les gens dans un cocon et les préserver de tous les risques » (responsable sport, C...).

Or, cette catégorisation, susceptible de faire émerger un « espace public pratique », s'est progressivement appuyée sur un véritable travail d'intelligibilité mené par les bailleurs sociaux, afin «d'améliorer » la gestion de leur parc. C'est, en effet, au moment où l'on est passé d'une gestion des flux de locataires à une gestion des stocks qui correspondait à un blocage des parcours résidentiels, que s'est développée une catégorisation institutionnelle essentiellement ethnique (Peraldi, op. cit.). Ce blocage a été provoqué notamment par la fin de « l'aide à la pierre » et de « l'embauche à salaire croissant » (Baudelot, Establet, 2000 : 82), dans un contexte marqué par les difficultés de la critique sociale (Boltanski, Chiapello, 1999 : 417). L'ethnicisation et la pathologisation des situations habitantes (familles « à problèmes », « polygames »...), ont servi de leurre, permettant aux bailleurs comme aux locataires, d'essentialiser les conséquences d'une situation socio-économique partagée par tous les publics. D'une part, cette question de la circulation à l'intérieur du parc est cruciale en période de blocage des parcours résidentiels. D'autre part, elle est motrice à l'égard des catégorisations à l'œuvre, parce que ces dernières assurent aux bailleurs une hiérarchisation de leur patrimoine par la rumeur et la stigmatisation que ne pourrait garantir le seul montant des loyers fortement encadré.

Nous sommes donc face à la conjonction d'intérêts objectifs entre d'une part, des bailleurs qui rendent leur patrimoine lisible par les réputations hiérarchisant des différentes opérations, et les catégorisations socio-ethniques qui contingentent les locataires ; et d'autre part, des habitants pour qui le logement doit rester, en période d'incertitude, un foyer essentiel de stabilité. La catégorisation devient alors centrale dans la construction identitaire des individus. Son caractère problématique pour les acteurs provient de la double dimension de ce travail de catégorisation, à la fois structurale et interactionnelle.

Les habitants travaillent en effet eux aussi cette mise en ordre par un travail au quotidien. Pour que la cité fonctionne comme une « région de significations ${ }^{6} \gg($ Joseph, 1998b : 119), il faut que la mémoire s'y articule au présent et rende les rôles connus et accessibles pour chacun, mais non inter-

6. Espace au sein duquel les rôles que peuvent endosser les individus sont facilement accessibles et les situations aisément appréhendables, parce que reconnaissables ou habituelles. 
changeables. A fortiori, lorsque la peur du trouble distingue le «nous » des « autres » et crée un « lien sécuritaire » (Anselme, $2000: 169)$. Nous allons voir que face au caractère problématique des catégorisations, les rassemblements de jeunes développent des types de relations sociales qui s'éloignent sensiblement de ce modèle de coproduction de catégories. Traversés par des tensions récurrentes, ils construisent en effet une « familiarité $a$ priori » qui paraît bien singulière au sein d'un tel environnement.

\section{DE LA CATÉgORISATION À LA « FAMILIARITÉ A PRIORI 》 : UN CHANGEMENT DE NATURE DES RELATIONS SOCIALES}

Les adolescents vivant dans les grands ensembles, pourtant longtemps réputés sans interstice, ont su y développer des avant-scènes et des « régions postérieures » permettant de «contredire sciemment l'impression produite par la représentation » (Goffman, $1973: 110)$.

« Je demande à un jeune pourquoi on ne le voit plus sur le rassemblement principal (situé à un carrefour), s'il y a une "embrouille" ou s'il a des problèmes. Il me répond : "C'est pas avec le quartier, c'est avec les flics" ».

Le rassemblement principal devient « trop » public mais fonctionne avec d'autres plus discrets.

Bosquets, parkings, jeux pour enfants, caves, aires de livraison, containers, friches urbaines..., le carnet de terrain donne en effet à voir une diversité bien éloignée de la caricature du hall squatté par des dealers. L'investissement de ces espaces a progressivement évolué dans un contexte marqué par des pratiques de précaution (portes blindées, sas, digicodes...) qui ont fermé l'espace, et par des pratiques de vigilance (caméras, agents de sécurité, éducateurs spécialisés...), mises en place pour veiller sur cet espace sécurisé :

« Le square va être déplacé vers la route pour que les policiers puissent le surveiller depuis leur voiture » (technicien espaces verts, B...).

«On est obligé d'appauvrir le paysage de la ville pour éviter de créer des lieux de rassemblement à problèmes. On nous demande de bien délimiter les lieux et les publics pour qu'il n'y ait pas d'interférence » (responsable espaces verts, B...).

La conception de cet espace, à la fois ouvert aux projets normés et fermé aux initiatives moins maîtrisables pour les gestionnaires, lui donne une dimension centrifuge. Un espace commun surexposé, qui ne permet ni l'anonymat, ni un filtrage interactionnel des relations, devient en effet invivable. Ce caractère centrifuge agit de manière évidente sur les locataires, qui stationnent peu sur l'espace commun résidentiel, mais il trouve son expression la plus paradoxale dans les rassemblements d'adolescents. Alors qu'il paraît centripète pour les observateurs extérieurs, le rassemblement est en effet centrifuge pour les jeunes qui le pratiquent. C'est le rassemblement qui donne la 
dynamique du mouvement de chacun, en procurant des motifs, des occasions, des circonstances, un compagnonnage... Bref, des situations :

« Nous, ça galérait beaucoup, mais tu avais l'impression que les gens croient que tous les jours, toute la journée j'étais là. Alors que non. J'allais en cours. Je rentrais. Je galérais un peu et puis après, je rentrais chez moi. J'avais des devoirs, des trucs comme ça. J'avais sport. Après, je revenais pour galérer. Et donc, en permanence, il y avait du monde » (Farid, 23 ans).

D'une part, cette pratique du rassemblement comme « embrayeur des mobilités », répond à la nécessité pour les adolescents de résister à une trop grande sédentarité, présentée comme une aliénation à la cité et coupant l'individu du reste de la vie sociale :

«Il est là dehors, mais il bouge pas avec eux. Il va pas au centre avec eux. Il va pas au café avec eux. Ce mec, il est bizarre. Très, très bizarre. Je te jure. Il doit avoir beaucoup de soucis. Il est malade. Il est asthmatique»(Faysal, 22 ans).

D'autre part, le mouvement s'y trouve valorisé parce que les pratiques, qui jouent des limites institutionnelles ou qui les traversent, favorisent les relations sociales réticulaires. Dans un réseau, c'est essentiellement l'engagement des individus dans chaque situation qui donne sa dimension publique à l'espace et aux sociabilités qui s'y produisent. Coproduite par le contexte et l'engagement des participants, l'urbanité d'une situation et d'un espace n'y est donc jamais garantie. Toujours relative, elle repose sur un travail interprétatif, facilité ou non par la disponibilité pratique des lieux et par notre capacité à rendre partageable nos engagements lors de ces situations :

" On jouait et souvent, on jouait devant le garage. Devant le bâtiment 9. On faisait des "deux-deux", "trois-trois" sur des petits espaces et les voitures, elles venaient au garage. Ça touchait et il y avait un monsieur, tu vois, il était assez impulsif. On le connaissait. Bon sinon, par sa fenêtre, il disait : "Arrêtez de faire du bruit" et tout ça. On discutait bien avec lui. Sympa, il nous appréciait. Et une fois, il arrive, ça jouait et la balle elle touche sa voiture. Il sort. Il commence à s'énerver et après il nous fait :

- "Il y a des stades pour jouer. Vous êtes tout le temps là".

C'est vrai qu'on était toujours dans le quartier.

- "Allez au stade Beuret. Pourquoi vous allez pas au stade Beuret ?"

- C'est quoi le stade Beuret?

- "Là-haut, là. Vous traversez Mille vent".

Après, on a connu ça. C'est le premier endroit. Et après, on allait tout le temps là » (Akim, 25 ans).

Les jeunes doivent en effet assumer les tensions pragmatiques liées à la nécessité de pouvoir militer pour une certaine conception des relations sociospatiales, tout en « habitant » dans un espace commun à partager avec autrui (Breviglieri, $2004: 6$ ) : 
« Lorsque les jeunes stationnent à l'extérieur du hall, ça se règle à l'amiable.

Quand c'est à l'intérieur, c'est beaucoup plus dur » (gardien d'immeuble, B...).

Au même titre que les squatters, ils résistent contre certains aspects du mode de vie urbain dominant (contraintes liées au travail salarié par exemple), portés par les adultes, les parents, ou encore les gestionnaires. Cette résistance s'opère depuis un mode d'hospitalité qui leur est propre, basé sur une «familiarité a priori» :

« Tu vois les jeunes, c'est comme une chaîne dont chacun serait un maillon. Il $\mathrm{y}$ a des maillons plus forts que d'autres » (Max, 20 ans).

$\mathrm{La}$ « forme » du rassemblement rend en effet familière a priori toute coprésence. S'il n'y a pas la volonté de connaître la vie privée des autres jeunes rassemblés, les sociabilités s'appuient sur le préalable tacite d'une probable histoire commune. Cette familiarité supposée s'étaye par la suite. Elle se confirme dans l'interaction. Notamment des nouveaux arrivants (la cooptation suffit), puisque la longue fréquentation des autres rassemblés, relativise l'ampleur et la préservation d'un espace intime. C'est parce que les rassemblements favorisent cette familiarité a priori que des affinités réticulaires se développent. Du coup, le caractère intermédiaire de cet espace croît, ainsi que sa dimension publique potentielle. Cette approche ne fait pas l'impasse sur la dimension parfois conflictuelle des rapports entre ces rassemblements et les publics qui les côtoient, mais doit au contraire nous amener à les réinterroger.

\section{DES CONFLITS AUX « ESPACES PUBLICS DE CONFRONTATION »}

En deçà de toute incivilité, une relation sociale implique « la jouissance commune de certains biens »(Goffman, op. cit., 68). Dans certaines relations, cette jouissance commune prend plus de place que dans d'autres. C'est, par exemple, ce qui permet au mari de fouiller dans le sac de sa femme et pas dans celui d'une autre. Il s'agit ici, à la fois d'une part commune, mais aussi d'une part de renoncement quant à la réserve habituellement en vigueur à l'égard des autres personnes. Pour Goffman, c'est la nature de ce renoncement, aux limites civiles notamment, qui mesure la « substance » de la relation. «La personne humaine est chose sacrée dont on ne doit pas violer les limites et, en même temps, toute relation implique d'abolir ces limites pour communiquer avec autrui » (Joseph, op. cit., 40). Une offense à l'égard de « règles de tact », qui sont habituellement respectées par les individus, est le premier signe d'une relation. Ce renoncement à la réserve habituelle suppose en effet une amorce de familiarité, un territoire commun, qui est donc préalable aux offenses. Alors qu'en apparence cette offense - la « dispute »porte justement sur des normes qui seraient mal partagées (les bonnes manières, la politesse...), c'est le fait de vivre dans le même espace ou d'être confronté aux mêmes épreuves qui permet progressivement de «prendre ses 
aises », de « se croire tout permis » ou de « manquer de respect », et qui exige de se différencier. Les jeunes qui composent les rassemblements éprouvent quotidiennement cette grande proximité socio-spatiale entre acteurs. Du coup, les relations sociales doivent se structurer dans ces intervalles réduits, tendues entre la menace du « trop » de distance (la coalition excluante, le préjugé...) et celle du «pas assez » où l'intimité se livre sans recul (la familiarité impudique, l'entre-soi ...). La discorde joue donc un rôle d'analyseur privilégié des rapports sociaux, culturels et territoriaux qui traversent les rassemblements.

Ces derniers sont fréquemment installés au carrefour de différents flux (au sens propre et au sens figuré) ce qui permet le plus souvent une pluralité de situations. De plus, l'aisance interactionnelle et la compétence spatiale de ceux qui y participent, confèrent aux rassemblements une évidente dimension publique. Cependant, d'une part, ces rassemblements se produisent le plus souvent sur des lieux ambigus, où le rôle symbolique et politique de l'espace le dispute à l'usage familiarisé qu'en font ces jeunes. Et c'est cette ambiguïté entre dimension politique commune (centre du quartier, entrée de la cité...) et usage (particulier), qui en fait un enjeu pour l'ensemble des publics. D'autre part, même quand les formes de l'interaction sont concertées (situation crédible, formatée par les usages, avec pour chacun un répertoire de rôles constitué), les jeunes qui se retrouvent quotidiennement sur l'espace commun des ensembles d'HLM, restent vulnérables, sous la pression d'une triple tension :

- leurs conduites doivent s'ajuster collectivement à des conventions, que pourtant la plupart de ces jeunes critiquent ;

- les activités du rassemblement doivent se réaliser au sein d'un environnement exposé (le rassemblement est visible quasiment en permanence, y compris lors de ses déplacements);

- chaque jeune doit pouvoir se socialiser de manière différentielle, lors de chaque situation à laquelle il est confronté : au lycée, en famille, au rassemblement, au travail...

« Ce qui est bizarre, c'est que moi au lycée, j'étais très agité. J'ai l'impression qu'à l'école, j'étais plus agité qu'au quartier. Je sais pas. Je pouvais pas supporter qu'on me parle n'importe comment. Et j'ai eu pas mal de conflits avec les profs. J'étais très impulsif. Adolescent, j'avais la rage. Peut-être contre toute forme d'autorité. La rage contre la police. Même les contrôleurs. [...], mais une fois que je suis rentré à la fac, je me suis calmé » (Akim, 25 ans).

Face à ces tensions, les modalisations ${ }^{7}$ nécessaires se manifestent par des signes d'appartenance qui s'éprouvent dans l'action avec l'utilisation des

7. Pour E. Goffman, une modalisation est un détournement partagé de l'action, qui permet aux acteurs de se l'approprier et de lui donner un sens à chaque fois renouvelé. 
surnoms, les façons de se serrer la main (le «tcheck»), ou même le poing sur le cœur qui suit le «tcheck» pour les musulmans et semble représenter autant un signe d'appartenance au «monde » des jeunes rassemblés qu'à la communauté des croyants. Ces modalisations s'expriment aussi sous forme d'allégeance des plus jeunes ou des plus faibles envers les plus âgés et les plus forts, ou encore par la valorisation de modèles :

« Ce gars-là, c'est un immense respect parce que, je trouve qu'il y est pour beaucoup. Les gens de sa génération, ils étaient quand même... ils commençaient à dévier, et lui, il fréquentait des gens beaucoup plus jeunes. Il nous a toujours orienté vers des bonnes choses, le sport [...]. Il fumait rien. Je te dis que ce gars là, pour moi, c'est un personnage essentiel. Je me suis beaucoup focalisé sur lui. C'était un modèle. C'était un modèle. J'ai rarement vu une personne comme ça. Incroyable. C'est incroyable. Pas d'insulte. Pas un mot au-dessus de l'autre. Pas d'embrouille » (Ali, 24 ans).

Inversement, la modalisation des interactions quotidiennes s'appuie aussi sur la dévalorisation de contre-modèles :

« Avant il y avait des meufs. Maintenant, ils vont avec des petites et si jamais ils peuvent les sauter, ils le font. Je suis pas un pédophile !» (Max, 20 ans).

Les « modalisations » visent à recadrer les situations et les réparations ont pour objectif de compenser les écarts commis. Elles se révèlent essentielles à partir du moment où l'on cherche à discerner la dimension publique des situations de tension quotidiennes. Anodines, c'est pourtant sur ces tensions que peut se construire un espace public pratique. Nous avons montré dans un article précédent (Boissonade, Devisme, 2005) comment épreuves et " cultures territoriales ${ }^{8}$ » jouent un rôle essentiel dans un passage vers une éventuelle mobilisation. Nous voudrions ici insister sur la nécessité de considérer la pluralité des pratiques de rassemblement comme des « espaces publics de confrontation » potentiels :

La salle principale de la MJC de B... est en pleine réfection. Les chaises ont donc été déménagées dans le hall d'entrée. Les jeunes, qui habituellement jouaient et discutaient dans un lieu à part, utilisent ces chaises disponibles installées dans le hall. Du coup, ils échangent avec les enfants du centre aéré, s'ajustent plus ou moins facilement avec les autres publics qui passent le seuil, ainsi qu'avec les responsables de l'institution. La confiance de chacun est mise à l'épreuve dans cette situation singulière, ce concours de circonstances ; mais c'est dans cette épreuve que l'espace public s'est créé, puis s'est éteint, une fois les chaises replacées dans la salle enfin repeinte.

8. Les cultures territoriales sont le recouvrement de deux champs : cultures et territoires. Elles prennent en compte les dimensions territoriales des cultures et les dimensions culturelles des territoires. 
Confrontés constamment à ce genre de situation fragile et non instituante, les jeunes rassemblés construisent une "familiarité a priori », dans laquelle les relations de confiance, permettent de rendre la position de chacun tenable. Face à ces " épreuves publiques ", ils doivent assurer une " gestion permanente du conflit potentiel »(Boissonade, op. cit.). La confiance n'est donc jamais totale. Il s'agit, le plus souvent, d'une confiance de « basse intensité », qui s'apparente à une « confiance de coordination ». Elle permet, par ajustements successifs, un passage du familier au collectif. Du coup, s'il veut être public, l'espace urbain doit gérer deux modèles contradictoires, celui du rapprochement et celui de la fuite. Accessible, il doit comporter une certaine rugosité sur laquelle s'appuyer, le cas échéant, tout en permettant un engagement ordinaire minimum. Évoquer une « hospitalité de confrontation » doit donc nous conduire à prendre la mesure de la discorde, même minime, sur laquelle se construit toute interaction. Nous avons vu que l'urbanité des rapports sociaux était relative aux situations plus qu'à des espaces particuliers, dits « publics », ou à des publics spécifiques, dits « urbains ». La figure des « rassemblements» de jeunes, plus que celle de groupe, nous a permis d'avancer l'idée d'un « espace public de confrontation ». Celui-ci repose notamment sur un type de sociabilité particulier aux rassemblements, la « familiarité a priori ». Elle prend appui sur des processus de catégorisation spécifiques et se développe sur toute une série d'espaces intermédiaires, loin de se cantonner aux seuls halls d'immeuble. Cette « urbanité de confrontation » met à l'épreuve les rassemblements et ceux qui les traversent. Elle pourrait se révéler utile pour apprécier les rapports de dépendance ou d'autonomie des liens sociaux par rapport aux lieux, bien au-delà d'ailleurs d'une problématique propre aux ensembles d'habitat social que nous venons d'aborder.

\section{RÉFÉRENCES BIBLIOGRAPHIQUES}

AnSELme, M. 2000. «Le lien sécuritaire ; mettre en ordre le désordre quotidien », dans M. Anselme (recueil), Du bruit à la parole, la scène politique des cités, La Tour d'Aigues, Éditions de l'Aube, p. 169-187.

Baudelot, C. ; Establet, R. 2000. Avoir 30 ans en 1968 et en 1998, Paris, Le Seuil. Boissonade, J. 2001. "Agrégations juvéniles et dynamiques du proche », Les Annales de la recherche urbaine, $\mathrm{n}^{\circ}$ 90, p. 173-181.

Boissonade, J. ; Devisme, L. 2005. « Mouvements et cultures territoriales », dans A. Bruston (sous la dir. de), Des cultures et des villes ; mémoires au futur, $\mathrm{La}$ Tour d'Aigues, Éditions de l'Aube, p. 133-150.

Boltanski, L. ; Chiapello, E. 1999. Le nouvel esprit du capitalisme, Paris, Gallimard.

BREVIGLIERI, M. 2004. « Le partage des propriétés. Circulation et empiètements dans les espaces communs », dans M. Breviglieri (sous la dir. de), Les choses dues. 
Propriétés, hospitalités et responsabilités; ethnographie des parties communes de squats militants, Paris, ministère de la Culture et de la Communication, Rapport à la Direction de l'architecture et du patrimoine, Mission du patrimoine ethnologique.

GofFman, E. 1973. La mise en scène de la vie quotidienne, La présentation de soi, tome 1, Paris, Éditions de Minuit.

HalbWaCHS, M. 1968. La mémoire collective, Paris, PUF.

JosEPH, I. 1998a. «L'accessibilité comme expérience sociale et interactionnelle », dans L. P. Grobois, I. Joseph et P. Sautet (sous la dir. de), Habiter une ville accessible; des usages à la conception, Paris, Éditions du MALTT, PCA.

Joseph, I. 1998b. La ville sans qualité, La Tour d'Aigues, Éditions de l'Aube.

MURARD, L. ; ZYLBERMAN, P. 1976. Le petit travailleur infatigable ou le prolétaire régénéré, Paris, Éditions Recherches.

PERAlDI, M. 1989. Entre logeurs et logés, épreuve territoriale, t. 2, Marseille, Cerfise.

Roulleau-Berger, L. 1991. La ville intervalle, jeunes entre centre et banlieue, Paris, Méridiens Klincksieck.

SchütZ, A.1987. Le chercheur et le quotidien. Phénoménologie des sciences sociales, Paris, Méridiens Klincksieck.

SIMMEL, G. 1988 [1909]. La tragédie de la culture, Paris, Rivages. 